\title{
FIXED POINT THEOREMS FOR NON-SELF MAPS I
}

\author{
TROY L. HICKS and LINDA MARIE SALIGA
}

Department of Mathematics and Statistics University of Missouri-Rolla

Rolla, MO 65401

(Received July 29, 1992)

\begin{abstract}
Suppose $f: C \rightarrow X$ where $C$ is a closed subset of $X$. Necessary and sufficient conditions are given for $f$ to have a fixed point. All results hold when $X$ is complete metric space. Several results hold in a much more general setting.
\end{abstract}

KEY WORDS AND PHRASES. Commuting, compatible, $d$-complete topological spaces, fixed points, non-self maps, pairs of mappings.

1991 AMS SUBJECT CLASSIFICATION CODE. 47H10, 54H25.

\section{INTRODUCTION.}

Fixed point theorems for non-self maps are unusual. We surely require that $C \cap f(C)$ is nonempty. $f(x)=x+1$ for $X$ in $[0,1]$ is a linear isometry from the compact space $[0,1]$ into the compact space $[0,2]$ but $f$ is fixed point free. The mapping $f(x)=x+\frac{1}{x}$ for $x$ in $[1, \infty)$ is a continuous mapping from $[1, \infty)$ into $[0, \infty)$. It is fixed point free but $|f(x)-f(y)|<|x-y|$ for $x \neq y$.

THEOREM (Brouwer [1]). If $E$ is a non-empty convex compact subset of $E^{n}$ and $f: E \rightarrow E$ is continuous, then $f(x)=x$ for some $x$ in $E$.

\section{RESULTS.}

THEOREM 1. Let $C$ be a closed subset of a complete metric space $X$ and suppose $f$ maps $C$ onto $X$ or $f$ maps $C$ into $X$ with $C \subset f(C)$. If for some $k>1, d(f(x), f(y)) \geq k d(x, y)$ for every $x, y$ in $C$, then $f$ has a unique fixed point in $C$.

PROOF. Clearly, $f$ is one-to-one. Let $g=f^{-1}$ restricted to $C$. Now $g$ maps $C$ into $C$. For $x, y$ in $C, d(x, y)=d(f(g x), f(g y)) \geq k d(g(x), g(y))$ or $d(g(x), g(y)) \leq \frac{1}{k} d(x, y)$ and $0<\frac{1}{k}<1 . g$ has a unique fixed point from Banach's fixed point theorem. But $f\left(x_{0}\right)=f\left(g\left(x_{0}\right)\right)=x_{0}$. If $x_{1}=f\left(x_{1}\right)$, then $g\left(x_{1}\right)=g\left(f\left(x_{1}\right)\right)=x_{1}$ and $c_{1}=x_{0}$.

The above result suggests that one should consider non-self maps that satisfy $C \subset f(C)$. It is well known that a continuous function from an arc onto a containing arc must have a fixed point. $[0,1]$ or any homeomorphic image is called an arc. Thus Brouwer's theorem extends to the case $C \subset f(C)$ for $n=1$. In [7], Sam Nadler showed that for $n \geq 2$ Brouwer's theorem does not extend. For $n \geq 2$, let $A$ and $B$ be closed balls in $E^{n}$ with $A \subset B$ and $A \neq B$. He showed that there exists $f$ and $g$ such that:

(a) $f: A \rightarrow B$ where $f$ is continuous, onto, $f(\partial A)=B$, and $f$ is fixed point free,

(b) $g: A \rightarrow B$ where $g$ is continuous, onto, $g^{-1}(\partial B)=\partial A$, and $g$ if fixed point free. 
THEOREM 2. Let $C$ be a closed bounded, and convex subset of a uniformly convex Banach space and suppose $f$ maps $C$ onto $X$ or $f$ maps $C$ into $X$ with $C \subset f(C)$. If for every $x, y$ in $C$ $\|f(x)-f(y)\| \geq\|x-y\|$, then $f$ has a fixed point in $C$.

PROOF. Clearly, $f$ is one-to-one. Let $g=f^{-1}$ restricted to $C$ and observe that $\|g(x)-g(y)\| \leq\|x-y\|$ where $g: C \rightarrow C$. From Kirk's theorem [6], $g$ has a fixed point $x_{0}$ in $C$. Clearly, $f\left(x_{0}\right)=x_{0}$.

The following is an example of a mapping $f$ that takes a closed, bounded, and convex subset $C$ of a Banach space $X$ into $X$ where $C \subset f(C),\|f(x)-f(y)\|=\|x-y\|$ for all $x, y \in C$, and $f$ has no fixed points.

EXAMPLE 1. Let $X$ be the space of sequences which converge to zero with $\|x\|=\sup _{n}\left|x_{n}\right|$ for $x$ in $X$. Let $C=\left\{x \in X:\|x\|=1\right.$ and $\left.x_{0}=1\right\} . C$ is closed, bounded, and convex. Define $f: C \rightarrow X$ by $f(x)=y$ where $y_{n}=x_{n+1}, n=0,1,2, \cdots\|f(x)-f(y)\|=\|x-y\|$ and $f$ is linear. To see that $C \subset f(C)$ consider the following. For $z \in C$, define $r$ to be the sequence where $r_{0}=1$ and $r_{n}=z_{n-1}, n=1,2,3, \cdots$. Then $r \in C$, and $f(r)=z$ so $C \subset f(C)$. If $s=\{1,0,0, \cdots\}, s \in C$ but $f(s)=\{0,0,0, \cdots\} \notin C$. Hence $C \neq f(C)$. If $f(x)=x$ for some $x$ in $C$, then $x_{n}=x_{n+1}$ for $n=0,1,2, \ldots$. Since $x_{0}=1, x_{n}=1$ for all $n$ and $x \notin C$. Therefore, $f$ does not have a fixed point in $C$.

The following example shows that Banach's fixed point theorem does not generalize to nonself maps.

EXAMPLE 2. Let $X=C(\boldsymbol{R}, \boldsymbol{R})$ with $\|f\|=\sup _{t \in \boldsymbol{R}}|f(t)|$ for $f \in X$. Let $C=\left\{f \in X: f(t)=0\right.$ for all $t \leq 0$ and $\left.\lim _{t \rightarrow \infty} f(t) \geq 1\right\} . \quad C$ is a closed and convex subset of $X$. Define $T: C \rightarrow X$ by $(T f)(t)=\frac{1}{2} f(t+1)$. To see that $C \subset T(C)$ consider the following. For $f$ in $C$ set $g(t)=2 f(t-1) . \quad g(t)=0$ for $t \leq 0$ since $t-1<0$ and $f(t)=0$ for all $t \leq 0$. $\operatorname{Lim}_{t \rightarrow \infty} g(t)=\lim _{t \rightarrow \infty} 2 f(t-1) \geq 2$. Thus $g \in C$ and $(T g)(t)=f(t)$. Hence $C \subset T(C)$. Let $f(t)$ be defined as 0 if $t \leq 0, t$ if $0<t<1$, and 1 if $t \geq 1$. Then $f \in C$. Now $(T f)(t)$ is 0 if $t \leq-1$, $\frac{1}{2}$ $(t+1)$ if $-1<t<0$, and $\frac{1}{2}$ if $t \geq 0$. Therefore, $T f \notin C$ and $C \neq T(C)$. For $f, g \in C,\|T f-T g\|=\frac{1}{2}\|f-g\|$. If $T f=f$ for some $f \in C$, then $f(t)=\frac{1}{2} f(t+1)$ and it follows that $f(n)=0$ for all integers $n$. Hence $\lim _{t \rightarrow \infty} f(t) \nsupseteq 1$ and $f \notin C$. Therefore $T$ does not have a fixed point in $C$. Note that $T$ is linear, one-to-one, and $T(C)$ is closed.

We now turn to finding necessary and sufficient conditions for a non-self map to have a fixed point. Then it becomes clear that $C \subset f(C)$ is a natural assumption.

Let $(X, t)$ be a topological space and $d: X \times X \rightarrow[0, \infty)$ such that $d(x, y)=0$ if and only if $x=y . \quad X$ is said to be $d$-complete if $\sum_{n=1}^{\infty} d\left(x_{n}, x_{n+1}\right)<\infty$ implies that the sequence $\left\{x_{n}\right\}$ is convergent in $(X, t)$. These spaces include complete (quasi) metric spaces and $d$-complete (symmetric) semi-metric spaces. In [2] and [3] several basic metric space fixed point theorems were extended to this setting. $f: X \rightarrow X$ is $w$-continuous at $x$ if $x_{n} \rightarrow x$ as $n \rightarrow \infty$ implies $f\left(x_{n}\right) \rightarrow f(x)$ as $n \rightarrow \infty$.

The following definition was given by G. Jungck in [5].

DEFINITION 1. Two maps $f$ and $g$ are compatible if, for any sequence $\left\{x_{n}\right\}$ such that $\lim _{n}$ $f\left(x_{n}\right)=\lim _{n} g\left(x_{n}\right)=t$ it follows that $\lim _{n} d\left(f\left(g x_{n}\right), g\left(f x_{n}\right)\right)=0$. Commuting maps are compatible but the converse is false.

DEFINITION 2. Given a map $f$, a map $g$ is compatible with $f$, if for any sequence $\left\{x_{n}\right\}$ such that $\lim _{n} f\left(x_{n}\right)=\lim _{n} g\left(x_{n}\right)=t$ it follows that $\lim _{n} f\left(g\left(x_{n}\right)\right)=g(t)$.

REMARK 1. If $f$ and $g$ are $w$-continuous and $(X, d)$ is a metric space, then, using definition 
$2, f$ is compatible with $g$ is equivalent to $g$ is compatible with $f$. In this case, we say that $f$ and $g$ are compatible.

PROOF. Assume $f$ and $g$ are $w$-continuous and that $\lim _{n} f\left(x_{n}\right)=\lim _{n} g\left(x_{n}\right)=t$ implies $\lim _{n}$ $f\left(g\left(x_{n}\right)\right)=g(t)$. If we are in a metric space,

and

$$
d\left(f\left(g x_{n}\right), g\left(f x_{n}\right)\right) \leq d\left(f\left(g x_{n}\right), g(t)\right)+d\left(g(t), g\left(f x_{n}\right)\right)
$$

$$
d\left(g\left(f x_{n}\right), f(t)\right) \leq d\left(g\left(f x_{n}\right), f\left(g x_{n}\right)\right)+d\left(f\left(g x_{n}\right), f(t)\right) .
$$

It follows that $f$ is compatible with $g$ implies that $g$ is compatible with $f$. Interchanging $g$ and $f$ above gives the converse.

It also follows from the above argument that if $f$ and $g$ are $w$-continuous and $(X, d)$ is a metric space, then the two definitions of compatibility are equivalent.

REMARK 2. If $(X, t)$ is a $d$-complete topological space, $g$ is $w$-continuous, and $f$ and $g$ commute, then $g$ is compatible with $f$ using definition 2 . We use definition 2 for $d$-complete topological spaces.

Theorem 3 and its corollaries are generalizations of theorems due to Hicks and Rhoades [4] which are generalizations of theorems due to Jungck [5].

THEOREM 3. Let $(X, t)$ be a Hausdorff $d$-complete topological space and suppose $f: C \rightarrow X$ where $f$ is $w$-continuous and $C$ is a closed subset of $X$. Then $f$ has a fixed point in $C$ if and only if there exists $\alpha \in(0,1)$ and a $w$-continuous function $g: C \rightarrow C$ such that $g(C) \subset f(C), g$ is compatible with $f$ on $f^{-1}(C)$ and

(1) $\quad d(g(x), g(y)) \leq \alpha d(f(x), f(y))$ for all $x, y \in C$.

Indeed, if (1) holds, $f$ and $g$ have a unique common fixed point.

PROOF. If $f(a)=a$ for some $a \in C$, set $g(x)=a$ for every $x \in C$. If $x \in f^{-1}(C), f(x) \in C$ and $g(f(x))=a=f(a)=f(g(x))$. If $\quad x \in C, g(x)=a=f(a)$ gives $g(C) \subset f(C)$. Also, $0=d(a, a)=d(g(x), g(y)) \leq \alpha d(f(x), f(y))$ for all $x, y \in C$.

Suppose there exists $\alpha \in(0,1)$ and a $w$-continuous function $g: C \rightarrow C$ such that $g(C) \subset f(C), g$ is compatible with $f$ on $f^{-1}(C)$ and $d(g(x), g(y)) \leq \alpha d(f(x), f(y))$ for all $x, y \in C$. Let $x_{0} \in C$. $g\left(x_{0}\right)=f\left(x_{1}\right)$ for some $x_{1} \in C$ since $g(C) \subset f(C)$. Construct a sequence $\left\{x_{n}\right\}$ with $\left\{x_{n}\right\} \subset C$ and $f\left(x_{n}\right)=g\left(x_{n-1}\right)$ for $n=1,2,3, \cdots$. Since

$$
d\left(f\left(x_{n}\right), f\left(x_{n+1}\right)\right)=d\left(g\left(x_{n-1}\right), g\left(x_{n}\right)\right) \leq \alpha d\left(f\left(x_{n-1}\right), f\left(x_{n}\right)\right),
$$

it follows that $d\left(f\left(x_{n}\right), f\left(x_{n+1}\right)\right) \leq \alpha^{n-1} d\left(f\left(x_{1}\right), f\left(x_{2}\right)\right)$. Hence $\sum_{n=1}^{\infty} d\left(f\left(x_{n}\right), f\left(x_{n+1}\right)\right)<\infty$. The space is $d$-complete so there exists $p \in X$ with $\lim _{n} f\left(x_{n}\right)=p$. $f\left(x_{n}\right)=g\left(x_{n-1}\right) \in C$ gives $p \in \operatorname{cl}(C)=C$. Now $f$ is $w$-continuous gives $f\left(g\left(x_{n-1}\right)\right) \rightarrow f(p)$ as $n \rightarrow \infty$. Since $g$ is compatible with $f$ on $f^{-1}(C)$ and $p \in f^{-1}(C)$ we get $\lim _{n} f\left(g\left(x_{n}\right)\right)=g(p)$. The space is Hausdorff so $f(p)=g(p)$ and $p \in f^{-1}(C)$. Consider the sequence $y_{n}=p$ for all $n$. Then $f\left(y_{n}\right) \rightarrow f(p)$ as $n \rightarrow \infty$, $g\left(y_{n}\right) \rightarrow g(p)$ as $n \rightarrow \infty$, and compatibility give $f(g(p))=f\left(g\left(y_{n}\right)\right) \rightarrow g(f(p))$ as $n \rightarrow \infty$. Thus, $f(g(p))=g(f(p)) . \quad$ Therefore, $\quad f(f(p))=f(g(p))=g(f(p))=g(g(p)) \quad$ together $\quad$ with $d(g(p), g(g p)) \leq \alpha d(f(p), f(g p)) \quad=\alpha d(g(p), g(g p)) \quad$ implies $\quad g(p)=g(g(p)) . \quad$ Hence $g(p)=g(g(p))=f(g(p))$ and $g(p)$ is a common fixed point of $f$ and $g$.

If $x=f(x)=g(x)$, then $\quad d(x, g(p))=d(g(x), g(g p)) \leq \alpha d(f(x), f(g p))=\alpha d(x, g(p)) \quad$ gives $x=g(p)$.

COROLLARY 1. Let $(X, t)$ be a Hausdorff $d$-complete topological space and $C$ be a closed 
subset of $X$. Suppose $f: C \rightarrow X$ and $g: C \rightarrow C$, where $f$ and $g$ are $w$-continuous, commute on $f^{-1}(C)$, and $g(C) \subset f(C)$. If there exists $\alpha \in(0,1)$ and a positive integer $k$ such that $d\left(g^{k}(x), g^{k}(y)\right) \leq \alpha d(f(x), f(y))$ for all $x, y \in C$, then $f$ and $g$ have a unique common fixed point.

PROOF. Clearly, $g^{k}$ commutes with $f$ on $f^{-1}(C)$ and $g^{k}(C) \subset g(C) \subset f(C)$. Applying the theorem to $g^{k}$ and $f$ gives a unique $p \in C$ such that $p=g^{k}(p)=f(p)$. Since $f$ and $g$ commute on $f^{-1}(C)$ and $p \in f^{-1}(C), g(p)=g(f(p))=f(g(p))=g^{k}(g(p))$ or $g(p)$ is a common fixed point of $f$ and $g^{k}$. Uniqueness of the common fixed point of $f$ and $g^{k}$ gives $g(p)=p=f(p)$. If $q=g(q)=f(q)$ then $g^{k}(q)=f(q)$ and $q=p$.

COROLLARY 2. Let $n$ be a positive integer and let $\alpha>1$. Suppose $C$ is a closed subset of a Hausdorff $d$-complete topological space and $f: C \rightarrow X$ with $C \subset f(C)$. If $d\left(f^{n}(x), f^{n}(y)\right)$ $\geq \alpha d(x, y)$ for all $x, y$ in $\left(f^{n-1}\right)^{-1}(C)$, then $f$ has a fixed point in $C$.

PROOF. For $n=1$, this follows from corollary 1 by letting $g=I . f^{n}$ is one-to-one. $C \subset f(C)$ implies $C \subset f^{n}(C)$. Let $h$ be the restriction of $\left(f^{n}\right)^{-1}$ to $C$. $h: C \rightarrow C$ and $d(h(x), h(y)) \leq \frac{1}{\alpha} d(x, y)$ for all $x, y \in C$. From corollary 1 with $k=1, h=g^{k}=g$ and $f=I$, there exists a unique $x_{0}$ such that $h\left(x_{0}\right)=x_{0}$. Hence $f\left(x_{0}\right)=f^{n+1}\left(x_{0}\right)=f^{n}\left(f\left(x_{0}\right)\right)$ or $h\left(f\left(x_{0}\right)\right)=\left(f^{n}\right)^{-1}\left(f\left(x_{0}\right)\right)=f\left(x_{0}\right)$. Uniqueness of the fixed point for $h$ gives $x_{0}=f\left(x_{0}\right)$. If $f(y)=y$ then $f^{n}(y)=y$ and $y=h(y)$. Again, uniqueness of the fixed point for $h$ gives $x_{0}=y$.

\section{REFERENCES}

1. BROUWER, L.E.J., Über abbildung von mannigfaltigkeiten, Math. Ann. 71 (1910), 97-115.

2. HICKS, T.L., Fixed point theorems for d-complete topological spaces I, Internat. J. Math. and Math. Sci. 15 (1992), 435-440.

3. HICKS, T.L. and RHOADES, B.E., Fixed point theorems for d-complete topological spaces II, Math. Japonica 37 (1992), 847-853.

4. HICKS, T.L. and RHOADES, B.E., Fixed points for pairs of mappings in d-complete topological spaces, Internat. J. Math. and Math. Sci. 16 (1993), 259-266.

5. JUNGCK, G., Compatible mappings and common fixed points, Internat. J. Math. and Math. Sci. 9 (1986), 771-779.

6. KIRK, W.A., A fixed point theorem for mappings which do not increase distance, Amer. Math. Monthly 72 (1965), 1004-1006.

7. NADLER, JR., S.B., Examples of fixed point free maps from cells onto larger cells and spheres, Rocky Mnt. J. Math. 11 (1981), 319-325. 


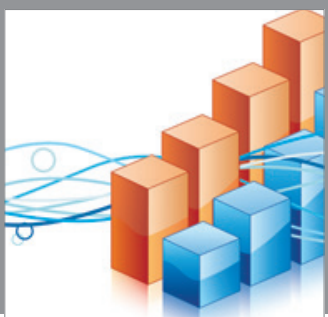

Advances in

Operations Research

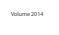

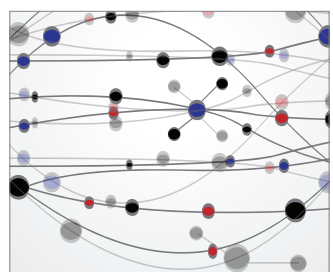

\section{The Scientific} World Journal
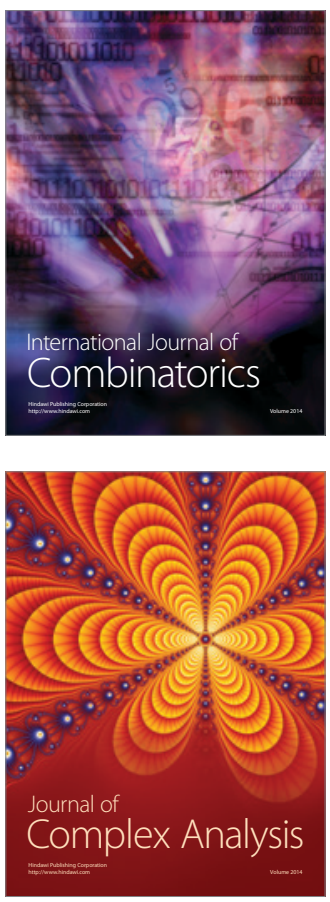

International Journal of

Mathematics and

Mathematical

Sciences
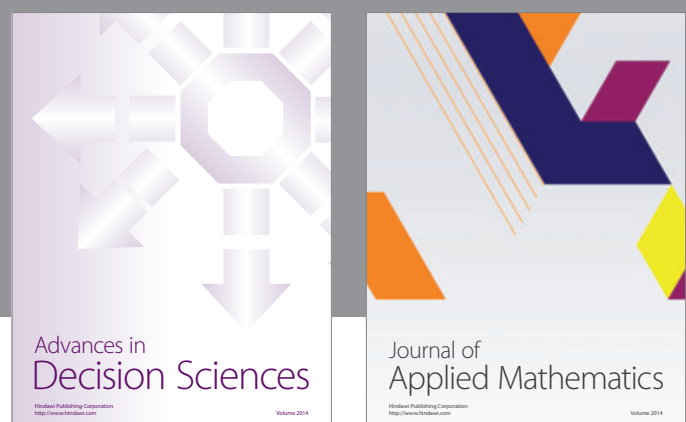

Journal of

Applied Mathematics
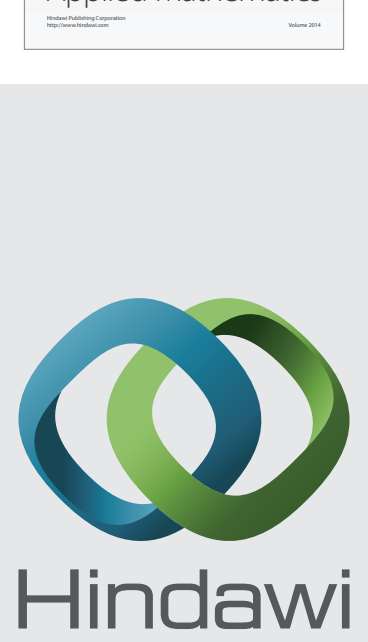

Submit your manuscripts at http://www.hindawi.com
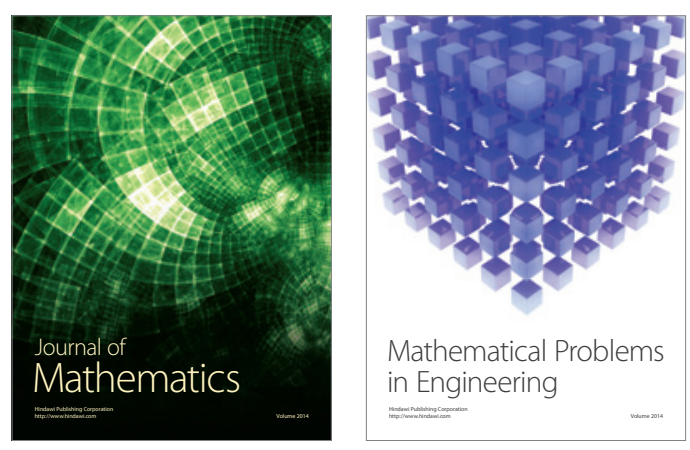

Mathematical Problems in Engineering
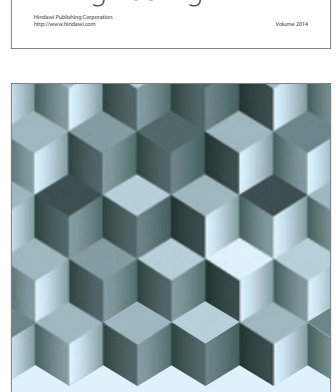

Journal of

Function Spaces
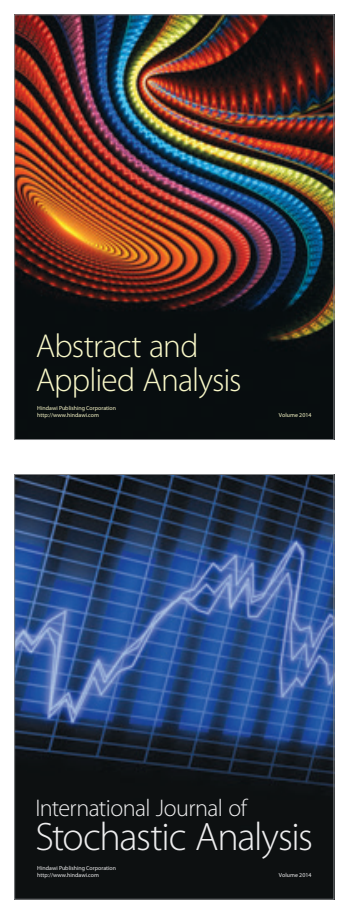

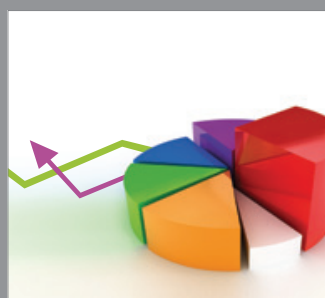

ournal of

Probability and Statistics

Promensencen
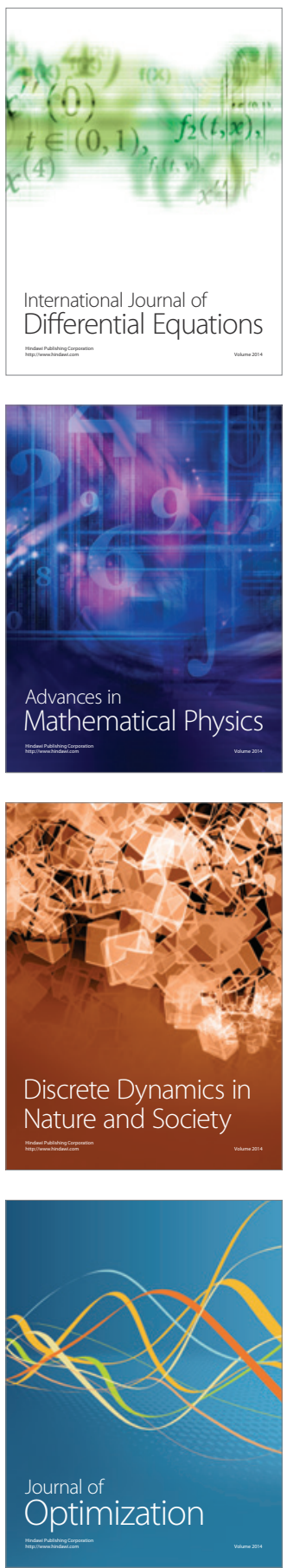